\title{
From Source Coding to MIMO - A Multi-Level Unequal Error Protection
}

\author{
Bashar Barmada \\ Department of Computer Science \\ Unitec Institute of Technology \\ Auckland, New Zealand \\ Email: bbarmada@unitec.ac.nz
}

\author{
Saeed Rehman \\ Department of Computer Science \\ Unitec Institute of Technology \\ Auckland, New Zealand \\ Email: srehman@unitec.ac.nz
}

\begin{abstract}
In this paper, unequal error protection (UEP) on different levels of the transmission system is proposed. Starting from the source coding level, two priority layers are produced, high priority (HP) and low priority (LP). At channel coding, each priority layer is turbo encoded with a coding rate that reflects its importance. For modulation, a more immune modulation mode is used to modulate the HP bit stream. Finally at the transmission level, a $3 \times 3$ MIMO is used, where more transmit antennas and time slots are offered to HP data. This arrangement of inequality over several levels increases the flexibility of the UEP system and leads to a better performance. Results show that the proposed system outperforms other semi-unequal and equal error protection systems over a wide range of channel signal-to-noise ratio (SNR), especially at low SNR values.
\end{abstract}

Keywords-Unequal error protection, Multi-layer source coding, Turbo coding, MIMO with spatial-time code

\section{INTRODUCTION}

Wireless communications continue to witness a rapid evolution driven by the increased number of users and the demand for higher data rate multimedia services. The future $5 \mathrm{G}$ mobile system is anticipated to achieve a capacity of 1 Gbps by 2020 . Advanced technologies at all levels of the transmission system become essential to support this vast amount of traffic, and at the same time, preserve reliability of the system [1], [2].

Unequal error protection (UEP) methods increase the reliability of systems by expanding the service over a wider range of channel signal-to-noise ratio (SNR) when compared to equal error protection (EEP) methods [3]. UEP can be implemented on several levels of the communication systems: on source coding level where many source layers with different importance are produced, on channel coding level using different coding rates for each priority layer, on modulation level with different modulation modes for the priority layers, and on transmission level using MIMO antennas, where better spatial channels are used for the important data. Different levels of the system can also be combined together for better UEP performance [4], [5], [6].

This paper proposes to combine the UEP techniques at all levels of the communications system (source coding, channel coding, modulation and MIMO) to exploit the advantages of each individual method and ease the constraints of the overall UEP system. For simplicity, only two priorities are considered; high priority (HP) and low priority (LP). However, the system can be scaled up to include higher number of priorities. Simulation results show that this combination of UEP methods on different levels of the system leads to a considerable improvement in the quality of the service over other semi-unequal and equal error protection systems. Next we describe the used UEP method at each of these levels.

\section{LEVELS OF UNEQUAL ERROR PROTECTION}

At source coding, JPEG2000 image coding is used as an example for its simplicity and because the coded bit stream can easily be organized into several layers with progression order. Each layer, when added, increases the quality of service progressively. For the proposed systems, two priorities layers are produced, HP and LP.

At channel coding, turbo code is used to offer the UEP for the two priority source layers with coding rates $R_{H}$ for HP and $R_{L}$ for LP, where $R_{H}<R_{L}$. This gives more redundancy to the most important layers, hence better correction-ability. In general, for $k$ source layers, the average coding rate of channel coding is given by:

$$
R_{a v g}=\frac{\sum_{i=1}^{k} p_{i}}{\sum_{i=1}^{k} \frac{p_{i}}{r_{i}}}
$$

where $p_{i}$ is the size of priority source layer $i$ and $r_{i}$ is the coding rate applied on priority layer $i$.

For modulation, 4-QAM (Quadrature Amplitude Modulation) is used to modulate HP bit stream with 2 bits per symbol, while 16-QAM is used to modulate LP bit stream with 4 bits per symbol. Although 16-QAM LP symbols have double of the data rate, they are more sensitive to channel errors and require more transmission power. After modulation the HP part occupies $33 \%$ of the overall bit stream and $66 \%$ goes to LP.

The size of the source layers produced at the source level are each adjusted according to its coding rate and modulation mode to maintain the right ratio of HP and LP in the overall bit stream, which will affect the overall quality of the service. The lower the coding rate, the better protection, but the overall quality of the service will be reduced. Figure 1 illustrates the percentage of redundancy added to each priority with different 


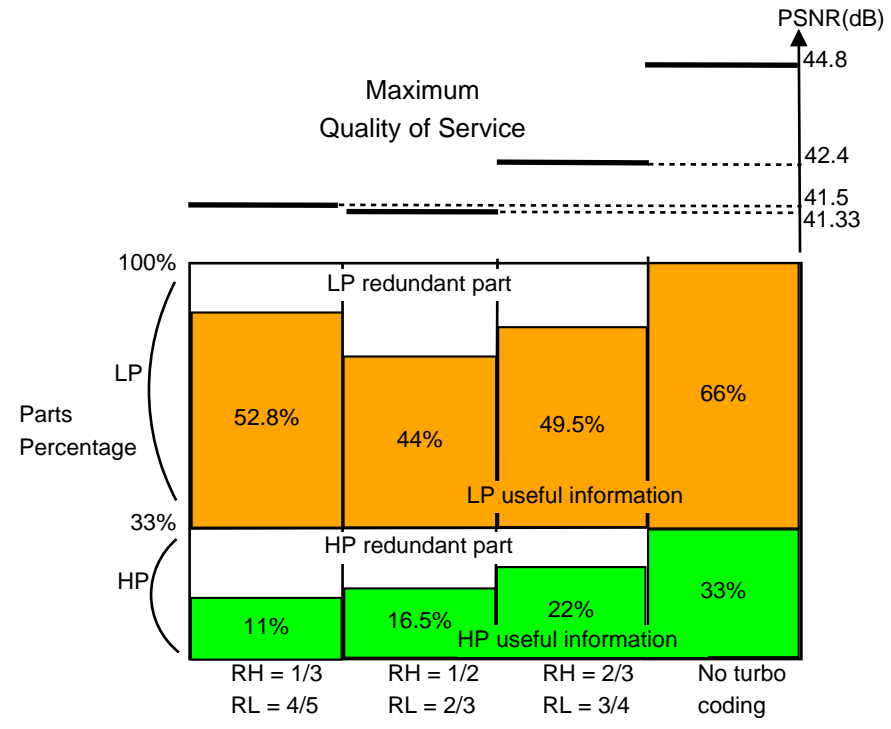

Fig. 1. The added redundancy by channel coding for HP and LP parts for different combinations of $R_{H}$ and $R_{L}$. The HP data is modulated with 4QAM while LP data is modulated with 16-QAM. The corresponding overall error-free PSNR is also shown for these combinations, for image JPEG2000 Lena $512 \times 512$

values of $\left(R_{H}, R_{L}\right)=(1 / 3,4 / 5),(1 / 2,2 / 3),(2 / 3,3 / 4)$. The figure shows also the reduction in the overall service quality for image Lena $512 \times 512$ coded at source coding ratio $2 \mathrm{bpp}$ and with layer progression order (SNR scalability).

Finally at the transmission level, the proposed system uses $3 \times 3$ MIMO antennas with two transmission slots. To achieve UEP more transmit antennas and more time slots are assigned to send the HP symbols. The orthogonal space-time block code (OSTBC) used in the system is based on Alamouti block code [7] and described in equation 2, where $S_{i, t}$ is the transmit symbol at transmit antenna $i$ and time slot $t$ ( $t 1$ or $t 2)$, which can be HP symbol $\left(S_{H P, t}\right)$ or LP symbol $\left(S_{L P, t}\right){ }^{*}$ is the complex conjugate of the symbol. In this code, the transmitted HP symbols gain a double protection compared to LP symbols.

$$
\left(\begin{array}{cc}
S_{1, t 1} & S_{1, t 2} \\
S_{2, t 1} & S_{2, t 2} \\
S_{3, t 1} & S_{3, t 2}
\end{array}\right)=\left(\begin{array}{cc}
S_{H P, t 1} & -S_{L P, t 2}^{*} \\
S_{L P, t 1} & S_{H P, t 2}^{*} \\
S_{H P, t 1} & S_{H P, t 2}^{*}
\end{array}\right)
$$

The system can be scaled easily to adapt more than three transmit / receive antennas to offer more varieties of UEP using MIMO. The price to pay here is the increase in complexity of the system.

\section{The Complete Mutli-LeVel UEP System}

Figure 2 shows the block diagram for the overall proposed UEP system with two priorities, where the inequality is offered through the different levels of the system, as described above.

At the receiver, each antenna receives three signals for each time slot, as the proposed system uses three transmit antennas. The signals at the receive antennas are given by equations in 3:

$$
\begin{array}{r}
R_{1, t 1}=h_{11} S_{H P, t}+h_{21} S_{L P, t 1}+h_{31} S_{H P, t 1}+n_{1, t 1} \\
R_{1, t 2}=-h_{11} S_{L P, t 2}^{*}+h_{21} S_{H P, t 2}^{*}+h_{31} S_{H P, t 2}^{*}+n_{1, t 2} \\
R_{2, t 1}=h_{12} S_{H P, t 1}+h_{22} S_{L P, t 1}+h_{32} S_{H P, t 1}+n_{2, t 1} \\
R_{2, t 2}=-h_{12} S_{L P, t 2}^{*}+h_{22} S_{H P, t 2}^{*}+h_{32} S_{H P, t 2}^{*}+n_{2, t 2} \\
R_{3, t 1}=h_{13} S_{H P, t 1}+h_{23} S_{L P, t 1}+h_{33} S_{H P, t 1}+n_{3, t 1} \\
R_{3, t 2}=-h_{13} S_{L P, t 2}^{*}+h_{23} S_{H P, t 2}^{*}+h_{33} S_{H P, t 2}^{*}+n_{3, t 2}
\end{array}
$$

where $R_{i, t}$ is the received signal at antenna $i$ and time slot t. $h_{j, i}$ is the channel response between transmit antenna $j$ and receive antenna $i$. All spatial channels are assumed to be independent and identically distributed (i.i.d), and the receiver knows the channel state information. $n_{i, t}$ is the Gaussian noise for the signal at the receive antenna $i$ and time slot $t$. The signal combiner in figure 2 extracts the HP and LP symbols from the received symbols for both time slots. Since the system uses non-binary QAM modulation, the combiner averages each priority parts together to produce the final received symbols for each priority $\left(Z_{H P}\right.$ and $\left.Z_{L P}\right)$, which form the input to the demodulation process, as described in equations 4 and 5.

$$
\begin{array}{r}
Z_{H P}=\operatorname{Avg}\left(h_{11}^{*} R_{1, t 1}, h_{31}^{*} R_{1, t 1},\right. \\
h_{21} R_{1, t 2}^{*}, h_{31} R_{1, t 2}^{*}, \\
h_{12}^{*} R_{2, t 1}, h_{32}^{*} R_{2, t 1}, \\
h_{22} R_{2, t 2}^{*}, h_{32} R_{2, t 2}^{*}, \\
h_{13}^{*} R_{3, t 1}, h_{33}^{*} R_{3, t 1}, \\
\left.h_{23} R_{3, t 2}^{*}, h_{33} R_{3, t 2}^{*}\right) \\
Z_{L P}=\operatorname{Avg}\left(h_{21}^{*} R_{1, t 1},-h_{11} R_{1, t 2}^{*},\right. \\
h_{22}^{*} R_{2, t 1},-h_{12} R_{2, t 2}^{*}, \\
\left.h_{23}^{*} R_{3, t 1},-h_{13} R_{3, t 2}^{*}\right)
\end{array}
$$

Figure 3 shows the BER and PSNR performance of the proposed UEP system in Raleigh flat fading channel for the combinations $\left(R_{H}, R_{L}\right)=(1 / 3,4 / 5),(1 / 2,2 / 3),(2 / 3,3 / 4)$. It is clear that the combination $(1 / 3,4 / 5)$ has the best performance for HP, but with the least quality, as its $R_{H}$ is the lowest, while is has the worst performance for LP part, as its $R_{L}$ is the highest. The difference between its HP and LP protection appears as a flat area in the PSNR performance. The other two combinations have less differences between HP and LP protection, and for that their PSNR performance increases more gradual when the channel SNR increases. Finding the right balance between $R_{H}$ and $R_{L}$ depends on the application and the quality of the channel that the service is using. For high quality channels it is more suitable to use high channel coding rates as they allow high overall service quality. For error prone channels it is more suitable to use low channel coding rates, as they guarantee the existence of the service with good quality.

The performance of the proposed multi-level UEP system when $\left(R_{H}, R_{L}\right)=(1 / 2,2 / 3)$ is compared to other semiUEP and EEP systems as described in table I. System A 


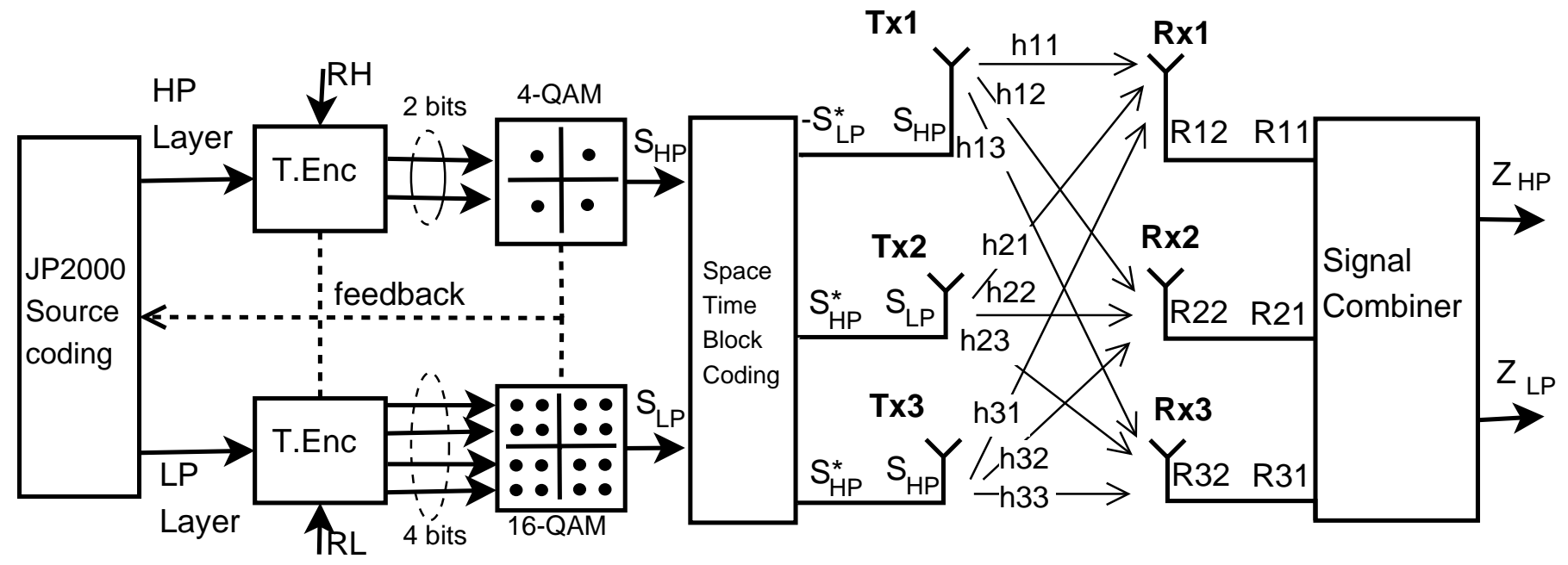

Fig. 2. The proposed Multi-level UEP system offers two priorities on different levels of the transmission system.

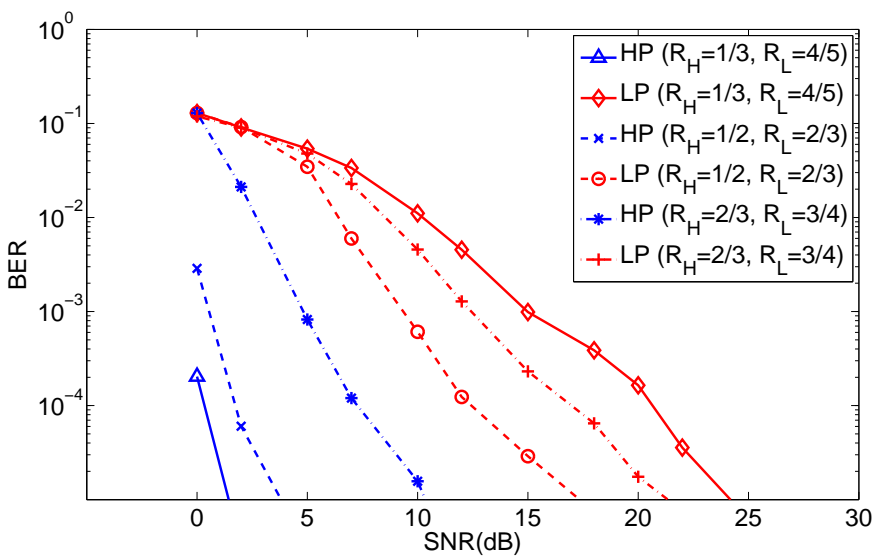

(a) BER

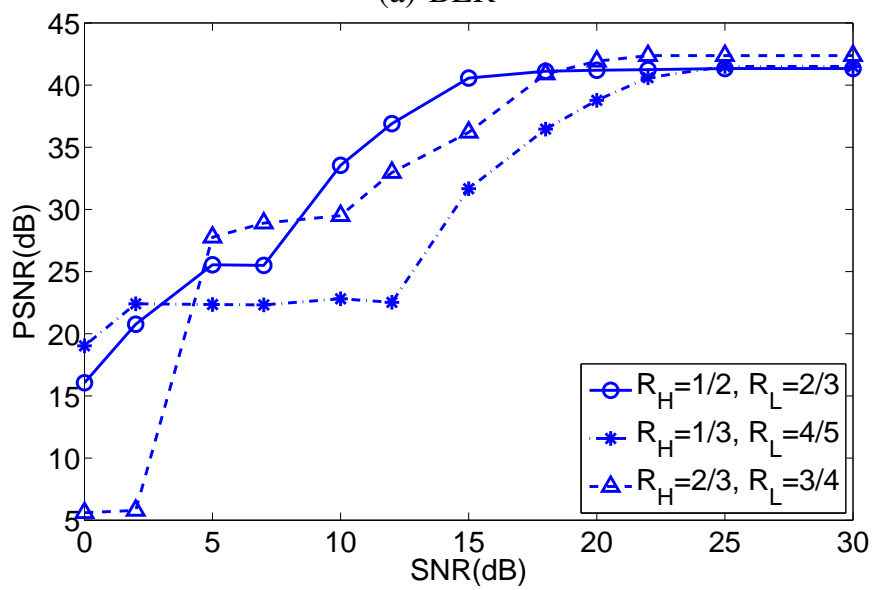

(b) PSNR

Fig. 3. The performance of the proposed multi-level UEP system in Rayleigh flat fading channel (a) BER, (b) PSNR for JPEG2000 image Lena $512 \times 512$.

differs from the proposed system only in the MIMO antennas
TABLE I

MULTI-LEVEL UEP SYSTEM VS. OTHER SEMI-UEP AND EEP SYSTEMS.

\begin{tabular}{|c|c|c|c|}
\hline & $\begin{array}{l}\text { Turbo } \\
\text { Coding }\end{array}$ & $\begin{array}{l}\text { Modulation } \\
\text { mode }\end{array}$ & $\begin{array}{l}\text { MIMO } \\
\text { System }\end{array}$ \\
\hline $\begin{array}{l}\text { Multi-level } \\
\text { UEP system }\end{array}$ & \multirow{3}{*}{$\begin{array}{l}R_{H}=1 / 2, \\
R_{L}=2 / 3\end{array}$} & $\begin{array}{l}\text { 4-QAM } \\
\text { (HP) }\end{array}$ & $\begin{array}{l}3 \times 3 \text { (HP has double } \\
\text { the protection of LP), } \\
\text { as in equation } 2\end{array}$ \\
\hline $\begin{array}{l}\text { System A } \\
\text { (semi-UEP) }\end{array}$ & & $\begin{array}{l}\text { 16-QAM } \\
\text { (LP) }\end{array}$ & $\left(\begin{array}{cc}S_{H P, t 1} & -S_{L P, t 2}^{*} \\
S_{L P, t 1} & S_{H P, t 2}^{*} \\
S_{H P, t 1} & S_{L P, t 2}^{*}\end{array}\right)$ \\
\hline $\begin{array}{l}\text { System B } \\
\text { (semi-UEP) }\end{array}$ & & 64-QAM & $\left(\begin{array}{cc}S_{t 1} & -S_{t 2}^{*} \\
S_{t 1} & S_{t 2}^{*}\end{array}\right)$ \\
\hline $\begin{array}{l}\text { System C } \\
\text { (EEP) }\end{array}$ & $\begin{array}{l}R_{\text {avg }}=3 / 5 \\
(\text { HP \& LP) }\end{array}$ & $\begin{array}{l}\text { (2 bits /HP, } \\
4 \text { bits /LP) }\end{array}$ & $\left.S_{t 2}^{*}\right)$ \\
\hline
\end{tabular}

where it uses equal resource distribution between HP and LP symbols. System B uses 64-QAM for modulation where each symbol carries 2 bits of HP and 4 bits of LP. The symbols then transmitted using $3 \times 3$ MIMO with spatial diversity to improve the reliability of the decision. System $\mathrm{C}$ differs only from system B in channel coding where the HP and LP bit streams are coded with the same coding rate $\left(R_{\text {avg }}=3 / 5\right)$, which is the average of coding rates $1 / 2$ and $2 / 3$ (according to equation 1). This makes system $\mathrm{C}$ a fully EEP one.

Figure 4 shows the PSNR performance in Rayleigh flat fading channel for the proposed multi-level UEP system together with the performance of the systems in table I. It is clear that the proposed system outperforms the others at low channel SNR values. After channel $\mathrm{SNR}=8 \mathrm{~dB}$, only system A shows slightly better performance over the proposed system when moving toward the final value of PSNR quality. This is because the LP symbols of system A have the same protection of HP symbols in the space-time code of MIMO antennas. 


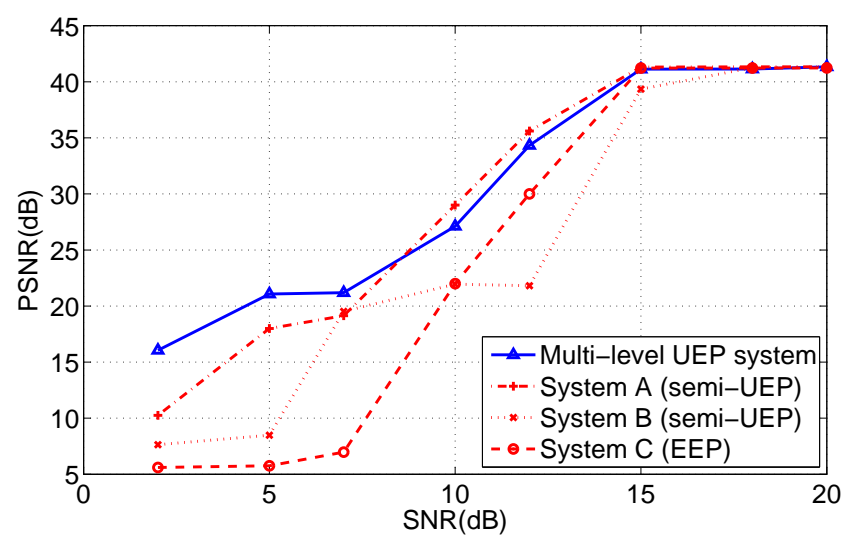

Fig. 4. PSNR performance in Rayleigh flat fading channel for the proposed multi-level UEP and other semi-UEP and EEP systems in table I, for JPEG2000 image Lena $512 \times 512$.

However, the improvement offered by the proposed system over system A at low channel SNR range is much noticeable compared to what system A offers at high channel SNRs.

System A itself outperforms systems B and C due to the better protection of its HP bit stream $\left(R_{H}=1 / 2\right.$ with 4 QAM). System B outperforms System C at low channel SNRs due to the better turbo coding rate for its HP bit stream. However, system C outperforms system B at high channel SNRs, as its LP bit stream has better turbo coding rate.

\section{CONCLUSION}

This paper proposed to use UEP on different levels of the transmission system. The proposed UEP system is demonstrated over two priorities. First, the JPEG2000 source encoder produces two priority layers HP and LP, where HP is more important than LP. Then turbo channel encoder protects each priority layer with a coding rate that suits its importance, where $R_{H}<R_{L}$. Next, the coded HP bit stream is modulated with 4-QAM and the coded LP bit stream is modulated with 16-QAM, as 4-QAM offers better immunity than 16-QAM against transmission errors, but carries half the capacity of 16-QAM. Finally, $3 \times 3$ MIMO antennas are adapted for transmission with two time slots. The used space-time block code offers more transmit antennas and time slots to the HP symbols. The performance of the proposed system when $\left(R_{H}, R_{L}\right)=(1 / 2,2 / 3)$ is compared to other semi-UEP and EEP systems (in table I). Results shows that the proposed system outperforms the others in Rayleigh flat fading channel at low channel SNRs, where the improvement is needed the most, and becomes second at high channel SNRs.

The proposed system offers a wide varieties of UEP options at different levels of the transmission system. Adjusting these UEP parameters will depend at the end on the used applications.

\section{REFERENCES}

[1] B. Bangerter, S. Talwar, R. Arefi, and K. Stewart, "Networks and devices for the 5g era," IEEE Communications Magazine, vol. 20, no. 2, pp. 90-96, 2014.
[2] N. Bhushan, J. Li, D. Malladi, R. Gilmore, D. Brenner, A. Damnjanovic, R. Sukhavasi, C. Patel, and S. Geirhofer, "Network densification: the dominant theme for wireless evolution into 5g," IEEE Communications Magazine, vol. 20, no. 2, pp. 82-89, 2014.

[3] B. Barmada, O. Baker, and A. Khoshnaw, "Unequal error protection for layered source coding using mimo technology," IET Electronics Letters, vol. 50, no. 4, pp. 325-327, 2014.

[4] J. Abot, C. Olivier, C. Perrine, and Y. Pousset, "A link adaptation scheme optimized for wireless jpeg2000 transmission over realistic mimo systems," Elsevier Signal Process: Image Communications, vol. 27, pp. 1066-1078, 2012.

[5] A. Vosoughi, P. Cosman, and L. Milstein, "Joint source-channel coding and unequal error protection for video plus depth," IEEE Signal Processing Letters, vol. 22, no. 1, pp. 31-34, 2015.

[6] H. Wang, C. Zhu, C. Xiong, and S. Chen, "Joint source-channel coding with unequal error protection using asymmetric turbo codes," in IEEE Transactions on Advanced Communications Technology (ICACT)) 15th International Conference, vol. 1, no. 1, 2013, pp. 1-10.

[7] S. Alamouti, "A simple transmit diversity technique for wireless communications," IEEE Select Areas in Communictions, vol. 16, no. 8, pp. 1451-1458, oct 1998. 